\title{
Epsilon Systems on Geometric Crystals of type $A_{n}^{\star}$
}

Toshiki NAKASHIMA

Department of Mathematics, Sophia University, 102-8554, Chiyoda-ku, Tokyo, Japan

E-mail: toshiki@mm.sophia.ac.jp

Received September 14, 2009, in final form January 28, 2010; Published online March 19, 2010

doi:10.3842/SIGMA.2010.023

\begin{abstract}
We introduce an epsilon system on a geometric crystal of type $A_{n}$, which is a certain set of rational functions with some nice properties. We shall show that it is equipped with a product structure and that it is invariant under the action of tropical $\mathrm{R}$ maps.
\end{abstract}

Key words: geometric crystal; epsilon system; tropical R map

2010 Mathematics Subject Classification: 17B37; 17B67; 22E65; 14M15

\section{Introduction}

In the theory of crystal bases, the piecewise-linear functions $\varepsilon_{i}$ and $\varphi_{i}$ play many crucial roles, e.g., description of highest weight vectors, tensor product of crystals, extremal vectors, etc. There exist counterparts for geometric crystals, denoted also $\left\{\varepsilon_{i}\right\}$, which are rational functions with several nice properties, indeed, they are needed to describe the product structure of geometric crystals (see Section 2) and in $\mathfrak{s l}_{2}$-case, the universal tropical $\mathrm{R}$ map is presented by using them [12].

In [1], higher objects $\varepsilon_{i, j}$ and $\varepsilon_{j, i}$ are introduced in order to prove the existence of product structure of geometric crystals induced from unipotent crystals, which satisfy the relation $\varepsilon_{i} \varepsilon_{j}=$ $\varepsilon_{i, j}+\varepsilon_{j, i}$ if the vertices $i$ and $j$ are simply laced. It motivates us to define further higher objects, "epsilon system".

The aim of the article is to define an "epsilon system" for type $A_{n}$ and reveal its basic properties, e.g., product structures and invariance under the action of tropical R maps. An epsilon system is a certain set of rational functions on a geometric crystal, which satisfy some relations with each other and have simple forms of the action by $e_{i}^{c}$ 's.

We found its prototype on the geometric crystal of the opposite Borel subgroup $B^{-} \subset$ $S L_{n+1}(\mathbb{C})$. In that case, indeed, the epsilon system is realized as a set of matrix elements and minor determinants of unipotent part of a group element in $B^{-}$(see Section 6). Therefore, we know that geometric crystals induced from unipotent crystals are equipped with an epsilon system naturally.

We shall introduce two remarkable properties of epsilon system: One is a product structure of epsilon systems. That is, for two geometric crystals with epsilon systems, say $\mathbb{X}$ and $\mathbb{Y}$, there exists canonically an epsilon system on the product of geometric crystals $\mathbb{X} \times \mathbb{Y}$ (see Section 5.3). The other is an invariance by tropical $\mathrm{R}$ maps: Let $\mathcal{R}: \mathbb{X} \times \mathbb{Y} \rightarrow \mathbb{Y} \times \mathbb{X}$ be a tropical $\mathrm{R}$ map (see Section 4) and $\varepsilon_{J}^{\mathbb{X} \times \mathbb{Y}}$ (resp. $\varepsilon_{J}^{\mathbb{Y} \times \mathbb{X}}$ ) an arbitrary element in the epsilon system on $\mathbb{X} \times \mathbb{Y}$ (resp. $\mathbb{Y} \times \mathbb{X}$ ) obtained from the ones on $\mathbb{X}$ and $\mathbb{Y}$. Then, we have the invariant property:

$$
\varepsilon_{J}^{\mathbb{Y} \times \mathbb{X}}(\mathcal{R}(x, y))=\varepsilon_{J}^{\mathbb{X} \times \mathbb{Y}}(x, y) .
$$

${ }^{\star}$ This paper is a contribution to the Proceedings of the Workshop "Geometric Aspects of Discrete and UltraDiscrete Integrable Systems" (March 30 - April 3, 2009, University of Glasgow, UK). The full collection is available at http://www.emis.de/journals/SIGMA/GADUDIS2009.html 
In the last section, we shall give an application of these results, which shows the uniqueness of tropical $\mathrm{R}$ map on some geometric crystals of type $A_{n}^{(1)}$.

Since the $\mathfrak{s l}_{2}$-universal tropical $\mathrm{R}$ map is presented by using the rational functions $\left\{\varepsilon_{i}\right\}$ as mentioned above, we expect that epsilon systems would be a key to find universal tropical $\mathrm{R}$ maps of higher ranks. For further aim, we would like to extend this notion to other simple Lie algebras, e.g., $B_{n}, C_{n}$, and $D_{n}$. These problems will be discussed elsewhere.

\section{Geometric crystals and unipotent crystals}

The notations and definitions here follow $[2,5,6,7,8,9,10,11]$.

\subsection{Geometric crystals}

Fix a symmetrizable generalized Cartan matrix $A=\left(a_{i j}\right)_{i, j \in I}$ with a finite index set $I$. Let $\left(\mathfrak{t},\left\{\alpha_{i}\right\}_{i \in I},\left\{h_{i}\right\}_{i \in I}\right)$ be the associated root data satisfying $\alpha_{j}\left(h_{i}\right)=a_{i j}$. Let $\mathfrak{g}=\mathfrak{g}(A)=$ $\left\langle\mathfrak{t}, e_{i}, f_{i}(i \in I)\right\rangle$ be the Kac-Moody Lie algebra associated with $A$ [5]. Let $P \subset \mathfrak{t}^{*}$ (resp. $Q:=\oplus_{i} \mathbb{Z} \alpha_{i}, Q^{\vee}:=\oplus_{i} \mathbb{Z} h_{i}$ ) be a weight (resp. root, coroot) lattice such that $\mathbb{C} \otimes P=\mathfrak{t}^{*}$ and $P \subset\left\{\lambda \mid \lambda\left(Q^{\vee}\right) \subset \mathbb{Z}\right\}$, whose element is called a weight.

Define the simple reflections $s_{i} \in \operatorname{Aut}(\mathfrak{t})(i \in I)$ by $s_{i}(h):=h-\alpha_{i}(h) h_{i}$, which generate the Weyl group $W$. Let $G$ be the Kac-Moody group associated with $(\mathfrak{g}, P)[6,7]$. Let $U_{\alpha}:=\exp \mathfrak{g}_{\alpha}$ ( $\alpha \in \Delta^{\text {re }}$ ) be the one-parameter subgroup of $G$. The group $G$ (resp. $U^{ \pm}$) is generated by $\left\{U_{\alpha} \mid \alpha \in \Delta^{\mathrm{re}}\right\}$ (resp. $\left\{U_{\alpha} \mid \alpha \in \Delta^{\mathrm{re}} \cap\left(\oplus_{i} \pm \mathbb{Z} \alpha_{i}\right)\right.$ ). Here $U^{ \pm}$is a unipotent subgroup of $G$. For any $i \in I$, there exists a unique group homomorphism $\phi_{i}: S L_{2}(\mathbb{C}) \rightarrow G$ such that

$$
\phi_{i}\left(\left(\begin{array}{ll}
1 & t \\
0 & 1
\end{array}\right)\right)=\exp \left(t e_{i}\right), \quad \phi_{i}\left(\left(\begin{array}{ll}
1 & 0 \\
t & 1
\end{array}\right)\right)=\exp \left(t f_{i}\right), \quad t \in \mathbb{C} .
$$

Set $\alpha_{i}^{\vee}(c):=\phi_{i}\left(\left(\begin{array}{cc}c & 0 \\ 0 & c^{-1}\end{array}\right)\right), x_{i}(t):=\exp \left(t e_{i}\right), y_{i}(t):=\exp \left(t f_{i}\right), G_{i}:=\phi_{i}\left(S L_{2}(\mathbb{C})\right), T_{i}:=\alpha_{i}^{\vee}\left(\mathbb{C}^{\times}\right)$ and $N_{i}:=N_{G_{i}}\left(T_{i}\right)$. Let $T$ be the subgroup of $G$ with $P$ as its weight lattice which is called a maximal torus in $G$, and let $B^{ \pm}(\supset T)$ be the Borel subgroup of $G$. We have the isomorphism $\phi: W \stackrel{\sim}{\longrightarrow} N / T$ defined by $\phi\left(s_{i}\right)=N_{i} T / T$. An element $\bar{s}_{i}:=x_{i}(-1) y_{i}(1) x_{i}(-1)$ is in $N_{G}(T)$, which is a representative of $s_{i} \in W=N_{G}(T) / T$.

Definition 2.1. Let $X$ be an ind-variety over $\mathbb{C}, \gamma_{i}$ and $\varepsilon_{i}(i \in I)$ rational functions on $X$, and $e_{i}: \mathbb{C}^{\times} \times X \rightarrow X$ a rational $\mathbb{C}^{\times}$-action. A quadruple $\left(X,\left\{e_{i}\right\}_{i \in I},\left\{\gamma_{i},\right\}_{i \in I},\left\{\varepsilon_{i}\right\}_{i \in I}\right)$ is a $G$ (or $\mathfrak{g})$-geometric crystal if

(i) $(\{1\} \times X) \cap \operatorname{dom}\left(e_{i}\right)$ is open dense in $\{1\} \times X$ for any $i \in I$, where $\operatorname{dom}\left(e_{i}\right)$ is the domain of definition of $e_{i}: \mathbb{C}^{\times} \times X \rightarrow X$.

(ii) The rational functions $\left\{\gamma_{i}\right\}_{i \in I}$ satisfy $\gamma_{j}\left(e_{i}^{c}(x)\right)=c^{a_{i j}} \gamma_{j}(x)$ for any $i, j \in I$.

(iii) $e_{i}$ and $e_{j}$ satisfy the following relations:

$$
\begin{array}{ll}
e_{i}^{c_{1}} e_{j}^{c_{2}}=e_{j}^{c_{2}} e_{i}^{c_{1}} & \text { if } a_{i j}=a_{j i}=0, \\
e_{i}^{c_{1}} e_{j}^{c_{1} c_{2}} e_{i}^{c_{2}}=e_{j}^{c_{2}} e_{i}^{c_{1} c_{2}} e_{j}^{c_{1}} & \text { if } a_{i j}=a_{j i}=-1, \\
e_{i}^{c_{1}} e_{j}^{c_{1}^{2} c_{2}} e_{i}^{c_{1} c_{2}} e_{j}^{c_{2}}=e_{j}^{c_{2}} e_{i}^{c_{1} c_{2}} e_{j}^{c_{1}^{2} c_{2}} e_{i}^{c_{1}} & \text { if } a_{i j}=-2, a_{j i}=-1, \\
e_{i}^{c_{1}} e_{j}^{c_{1}^{3} c_{2}} e_{i}^{c_{1}^{2} c_{2}} e_{j}^{c_{1}^{3} c_{2}^{2}} e_{i}^{c_{1} c_{2}} e_{j}^{c_{2}}=e_{j}^{c_{2}} e_{i}^{c_{1} c_{2}} e_{j}^{c_{1}^{3} c_{2}^{2}} e_{i}^{c_{1}^{2} c_{2}} e_{j}^{c_{1}^{3} c_{2}} e_{i}^{c_{1}} & \text { if } a_{i j}=-3, a_{j i}=-1 .
\end{array}
$$

(iv) The rational functions $\left\{\varepsilon_{i}\right\}_{i \in I}$ satisfy $\varepsilon_{i}\left(e_{i}^{c}(x)\right)=c^{-1} \varepsilon_{i}(x)$ and $\varepsilon_{i}\left(e_{j}^{c}(x)\right)=\varepsilon_{i}(x)$ if $a_{i, j}=$ $a_{j, i}=0$. 
The relations in (iii) is called Verma relations. If $\chi=\left(X,\left\{e_{i}\right\},\left\{\gamma_{i}\right\},\left\{\varepsilon_{i}\right\}\right)$ satisfies the conditions (i), (ii) and (iv), we call $\chi$ a pre-geometric crystal.

Remark. The last condition (iv) is slightly modified from $[2,9,10,11,12]$ since all $\varepsilon_{i}$ appearing in these references satisfy the new condition ' $\varepsilon_{i}\left(e_{j}^{c}(x)\right)=\varepsilon_{i}(x)$ if $a_{i, j}=a_{j, i}=0$ ' and we need this condition to define "epsilon systems" later.

\subsection{Unipotent crystals}

In the sequel, we denote the unipotent subgroup $U^{+}$by $U$. We define unipotent crystals (see $[1,9])$ associated to Kac-Moody groups.

Definition 2.2. Let $X$ be an ind-variety over $\mathbb{C}$ and $\alpha: U \times X \rightarrow X$ be a rational $U$-action such that $\alpha$ is defined on $\{e\} \times X$. Then, the pair $\mathbf{X}=(X, \alpha)$ is called a $U$-variety. For $U$-varieties $\mathbf{X}=\left(X, \alpha_{X}\right)$ and $\mathbf{Y}=\left(Y, \alpha_{Y}\right)$, a rational map $f: X \rightarrow Y$ is called a $U$-morphism if it commutes with the action of $U$.

Now, we define a $U$-variety structure on $B^{-}=U^{-} T$. As in [8], the Borel subgroup $B^{-}$is an ind-subgroup of $G$ and hence an ind-variety over $\mathbb{C}$. The multiplication map in $G$ induces the open embedding; $B^{-} \times U \hookrightarrow G$, which is a birational map. Let us denote the inverse birational map by $g: G \longrightarrow B^{-} \times U$ and let rational maps $\pi^{-}: G \rightarrow B^{-}$and $\pi: G \rightarrow U$ be $\pi^{-}:=\operatorname{proj}_{B^{-}} \circ g$ and $\pi:=\operatorname{proj}_{U} \circ g$. Now we define the rational $U$-action $\alpha_{B^{-}}$on $B^{-}$by

$$
\alpha_{B^{-}}:=\pi^{-} \circ m: U \times B^{-} \longrightarrow B^{-},
$$

where $m$ is the multiplication map in $G$. Then we get $U$-variety $\mathbf{B}^{-}=\left(B^{-}, \alpha_{B^{-}}\right)$.

\section{Definition 2.3.}

(i) Let $\mathbf{X}=(X, \alpha)$ be a $U$-variety and $f: X \rightarrow B^{-}$a $U$-morphism. The pair $(\mathbf{X}, f)$ is called a unipotent $G$-crystal or, for short, unipotent crystal.

(ii) Let $\left(\mathbf{X}, f_{X}\right)$ and $\left(\mathbf{Y}, f_{Y}\right)$ be unipotent crystals. A $U$-morphism $g: \mathbf{X} \rightarrow \mathbf{Y}$ is called a morphism of unipotent crystals if $f_{X}=f_{Y} \circ g$. In particular, if $g$ is a birational map of ind-varieties, it is called an isomorphism of unipotent crystals.

We define a product of unipotent crystals following [1]. For unipotent crystals $\left(\mathbf{X}, f_{X}\right)$, $\left(\mathbf{Y}, f_{Y}\right)$, define a rational map $\alpha_{X \times Y}: U \times X \times Y \rightarrow X \times Y$ by

$$
\alpha_{X \times Y}(u, x, y):=\left(\alpha_{X}(u, x), \alpha_{Y}\left(\pi\left(u \cdot f_{X}(x)\right), y\right)\right) .
$$

\section{Theorem $2.4([1])$.}

(i) The rational map $\alpha_{X \times Y}$ defined above is a rational $U$-action on $X \times Y$.

(ii) Let $\mathbf{m}: B^{-} \times B^{-} \rightarrow B^{-}$be a multiplication map and $f=f_{X \times Y}: X \times Y \rightarrow B^{-}$be the rational map defined by

$$
f_{X \times Y}:=\mathbf{m} \circ\left(f_{X} \times f_{Y}\right) .
$$

Then $f_{X \times Y}$ is a $U$-morphism and $\left(\mathbf{X} \times \mathbf{Y}, f_{X \times Y}\right)$ is a unipotent crystal, which we call a product of unipotent crystals $\left(\mathbf{X}, f_{X}\right)$ and $\left(\mathbf{Y}, f_{Y}\right)$.

(iii) Product of unipotent crystals is associative. 


\subsection{From unipotent crystals to geometric crystals}

For $i \in I$, set $U_{i}^{ \pm}:=U^{ \pm} \cap \bar{s}_{i} U^{\mp} \bar{s}_{i}^{-1}$ and $U_{ \pm}^{i}:=U^{ \pm} \cap \bar{s}_{i} U^{ \pm} \bar{s}_{i}^{-1}$. Indeed, $U_{i}^{ \pm}=U_{ \pm \alpha_{i}}$. Set

$$
Y_{ \pm \alpha_{i}}:=\left\langle x_{ \pm \alpha_{i}}(t) U_{\alpha} x_{ \pm \alpha_{i}}(-t) \mid t \in \mathbb{C}, \alpha \in \Delta_{ \pm}^{\mathrm{re}} \backslash\left\{ \pm \alpha_{i}\right\}\right\rangle \text {, }
$$

where $x_{\alpha_{i}}(t):=x_{i}(t)$ and $x_{-\alpha_{i}}(t):=y_{i}(t)$. We have the unique decomposition;

$$
U^{-}=U_{i}^{-} \cdot Y_{ \pm \alpha_{i}}=U_{-\alpha_{i}} \cdot U_{-}^{i} .
$$

By using this decomposition, we get the canonical projection $\xi_{i}: U^{-} \rightarrow U_{-\alpha_{i}}$. Now, we define the function on $U^{-}$by

$$
\chi_{i}:=y_{i}^{-1} \circ \xi_{i}: U^{-} \longrightarrow U_{-\alpha_{i}} \stackrel{\sim}{\longrightarrow} \mathbb{C},
$$

and extend this to the function on $B^{-}$by $\chi_{i}(u \cdot t):=\chi_{i}(u)$ for $u \in U^{-}$and $t \in T$. For a unipotent $G$-crystal $\left(\mathbf{X}, f_{X}\right)$, we define a function $\varepsilon_{i}:=\varepsilon_{i}^{X}: X \rightarrow \mathbb{C}$ by

$$
\varepsilon_{i}:=\chi_{i} \circ f_{X},
$$

and a rational function $\gamma_{i}: X \rightarrow \mathbb{C}$ by

$$
\gamma_{i}:=\alpha_{i} \circ \operatorname{proj}_{T} \circ f_{X}: X \rightarrow B^{-} \rightarrow T \rightarrow \mathbb{C},
$$

where $\operatorname{proj}_{T}$ is the canonical projection.

Remark. Note that the function $\varepsilon_{i}$ is denoted by $\varphi_{i}$ in $[1,9]$.

Suppose that the function $\varepsilon_{i}$ is not identically zero on $X$. We define a morphism $e_{i}$ : $\mathbb{C}^{\times} \times X \rightarrow X$ by

$$
e_{i}^{c}(x):=x_{i}\left(\frac{c-1}{\varepsilon_{i}(x)}\right)(x)
$$

Theorem 2.5 ([1]). For a unipotent $G$-crystal $\left(\mathbf{X}, f_{X}\right)$, suppose that the function $\varepsilon_{i}$ is not identically zero for any $i \in I$. Then the rational functions $\gamma_{i}, \varepsilon_{i}: X \rightarrow \mathbb{C}$ and $e_{i}: \mathbb{C}^{\times} \times X \rightarrow X$ as above define a geometric $G$-crystal $\left(\mathbf{X},\left\{e_{i}\right\}_{i \in I},\left\{\gamma_{i}\right\}_{i \in I},\left\{\varepsilon_{i}\right\}_{i \in I}\right)$, which is called the induced geometric $G$-crystals by unipotent $G$-crystal $\left(\mathbf{X}, f_{X}\right)$.

Proposition 2.6. For unipotent $G$-crystals $\left(\mathbf{X}, f_{X}\right)$ and $\left(\mathbf{Y}, f_{Y}\right)$, set the product $\left(\mathbf{Z}, f_{Z}\right):=$ $\left(\mathbf{X}, f_{X}\right) \times\left(\mathbf{Y}, f_{Y}\right)$, where $Z=X \times Y$. Let $\left(Z,\left\{e_{i}^{Z}\right\}_{i \in I},\left\{\gamma_{i}^{Z}\right\}_{i \in I},\left\{\varepsilon_{i}^{Z}\right\}_{i \in I}\right)$ be the induced geometric $G$-crystal from $\left(\mathbf{Z}, f_{Z}\right)$. Then we obtain:

(i) For each $i \in I,(x, y) \in Z$,

$$
\gamma_{i}^{Z}(x, y)=\gamma_{i}^{X}(x) \gamma_{i}^{Y}(y), \quad \varepsilon_{i}^{Z}(x, y)=\varepsilon_{i}^{X}(x)+\frac{\varepsilon_{i}^{Y}(y)}{\gamma_{i}^{X}(x)}
$$

(ii) For any $i \in I$, the action $e_{i}^{Z}: \mathbb{C}^{\times} \times Z \rightarrow Z$ is given by: $\left(e_{i}^{Z}\right)^{c}(x, y)=\left(\left(e_{i}^{X}\right)^{c_{1}}(x),\left(e_{i}^{Y}\right)^{c_{2}}(y)\right)$, where

$$
c_{1}=\frac{c \gamma_{i}^{X}(x) \varepsilon_{i}^{X}(x)+\varepsilon_{i}^{Y}(y)}{\gamma_{i}^{X}(x) \varepsilon_{i}^{X}(x)+\varepsilon_{i}^{Y}(y)}, \quad c_{2}=\frac{c\left(\gamma_{i}^{X}(x) \varepsilon_{i}^{X}(x)+\varepsilon_{i}^{Y}(y)\right)}{c \gamma_{i}^{X}(x) \varepsilon_{i}^{X}(x)+\varepsilon_{i}^{Y}(y)} .
$$

Here note that $c_{1} c_{2}=c$. The formula $c_{1}$ and $c_{2}$ in [1] seem to be different from ours. 


\section{Prehomogeneous geometric crystal}

Definition 3.1. Let $\chi=\left(X,\left\{e_{i}^{c}\right\},\left\{\gamma_{i}\right\},\left\{\varepsilon_{i}\right\}\right)$ be a geometric crystal. We say that $\chi$ is prehomogeneous if there exists a Zariski open dense subset $\Omega \subset X$ which is an orbit by the actions of the $e_{i}^{c}$ 's.

Lemma $3.2([3])$. Let $\chi_{j}=\left(X_{j},\left\{e_{i}^{c}\right\},\left\{\gamma_{i}\right\},\left\{\varepsilon_{i}\right\}\right)(j=1,2)$ be prehomogeneous geometric crystals. Let $\Omega_{1} \subset X_{1}$ be an open dense orbit in $X_{1}$. For isomorphisms of geometric crystals $\phi, \phi^{\prime}: \chi_{1} \rightarrow \chi_{2}$, suppose that there exists $p_{1} \in \Omega_{1}$ such that $\phi\left(p_{1}\right)=\phi^{\prime}\left(p_{1}\right) \in X_{2}$. Then, we have $\phi=\phi^{\prime}$ as rational maps.

Theorem 3.3 ([3]). Let $\chi=\left(X,\left\{e_{i}^{c}\right\},\left\{\gamma_{i}\right\},\left\{\varepsilon_{i}\right\}\right)$ be a finite-dimensional positive geometric crystal with the positive structure $\theta:\left(\mathbb{C}^{\times}\right)^{\operatorname{dim}(X)} \rightarrow X$ and $B:=\mathcal{U} D_{\theta}(\chi)$ the crystal obtained as the ultra-discretization of $\chi$. If $B$ is a connected crystal, then $\chi$ is prehomogeneous.

In $[2,3]$, we showed that ultra-discretization of the affine geometric crystal $\mathcal{V}(\mathfrak{g})_{l}(l>0)$ is a limit of perfect crystal $B_{\infty}\left(\mathfrak{g}^{L}\right)$, where $\mathfrak{g}^{L}$ is the Langlands dual of $\mathfrak{g}$. Since for any $k \in$ $\mathbb{Z}_{>0}$ a tensor product $B_{\infty}\left(\mathfrak{g}^{L}\right)^{\otimes k}$ is connected by the perfectness of $B_{\infty}\left(\mathfrak{g}^{L}\right)$ and we have the isomorphism of crystals

$$
\mathcal{U} D\left(\mathcal{V}(\mathfrak{g})_{L_{1}} \times \cdots \times \mathcal{V}(\mathfrak{g})_{L_{k}}\right) \cong B_{\infty}\left(\mathfrak{g}^{L}\right)^{\otimes k}, \quad L_{1}, \ldots, L_{k}>0,
$$

by Theorem 3.3 we obtain the following:

Corollary $3.4([3]) . \mathcal{V}(\mathfrak{g})_{L_{1}} \times \cdots \times \mathcal{V}(\mathfrak{g})_{L_{k}}$ is prehomogeneous.

\section{Tropical $\mathrm{R}$ maps}

Definition 4.1. Let $\left\{X_{\lambda}\right\}_{\lambda \in \Lambda}$ be a family of geometric crystals with the product structures, where $\Lambda$ is an index set. A birational map $\mathcal{R}_{\lambda \mu}: X_{\lambda} \times X_{\mu} \longrightarrow X_{\mu} \times X_{\lambda}$ is called a tropical $R$ map if they satisfy:

$$
\begin{aligned}
& \left(e_{i}^{X_{\mu} \times X_{\lambda}}\right)^{c} \circ \mathcal{R}_{\lambda \mu}=\mathcal{R}_{\lambda \mu} \circ\left(e_{i}^{X_{\lambda} \times X_{\mu}}\right)^{c}, \\
& \varepsilon_{i}^{X_{\lambda} \times X_{\mu}}=\varepsilon_{i}^{X_{\mu} \times X_{\lambda}} \circ \mathcal{R}_{\lambda \mu} \\
& \gamma_{i}^{X_{\lambda} \times X_{\mu}}=\gamma_{i}^{X_{\mu} \times X_{\lambda}} \circ \mathcal{R}_{\lambda \mu} \\
& \mathcal{R}_{\lambda \mu} \mathcal{R}_{\mu \nu} \mathcal{R}_{\lambda \mu}=\mathcal{R}_{\mu \nu} \mathcal{R}_{\lambda \mu} \mathcal{R}_{\mu \nu}
\end{aligned}
$$

for any $i \in I$ and $\lambda, \mu, \nu \in \Lambda$.

Tropical R maps for certain affine geometric crystals of type $A_{n}^{(1)}, B_{n}^{(1)}, D_{n}^{(1)}, D_{n+1}^{(2)}, A_{2 n-1}^{(2)}$ and $A_{2 n}^{(2)}$ are described explicitly [3, 4].

The following is immediate from Lemma 3.2 and Corollary 3.4.

Theorem $4.2([3])$. Let $\mathcal{R}, \mathcal{R}^{\prime}: \mathcal{V}_{L} \times \mathcal{V}_{M} \rightarrow \mathcal{V}_{M} \times \mathcal{V}_{L}$ be tropical $R$ maps. Suppose that there exists $p \in \mathcal{V}_{L} \times \mathcal{V}_{M}$ such that $\mathcal{R}(p)=\mathcal{R}^{\prime}(p)$. Then we have $\mathcal{R}=\mathcal{R}^{\prime}$ as birational maps.

Let us introduce an example of a tropical R map of type $A_{n}^{(1)}$.

Example 4.3. Set $\mathcal{B}_{L}:=\left\{l=\left(l_{1}, \ldots, l_{n+1}\right) \mid l_{1} l_{2} \cdots l_{n+1}=L\right\}$, which is equipped with an $A_{n}^{(1)}$-geometric crystal structure by:

$$
e_{i}^{c}(l)=\left(\ldots, c l_{i}, c^{-1} l_{i+1}, \ldots\right), \quad \gamma_{i}(l)=l_{i} / l_{i+1}, \quad \varepsilon_{i}(l)=l_{i+1}, \quad i=0,1, \ldots, n .
$$


The tropical $\mathrm{R}$ map on $\left\{\mathcal{B}_{L}\right\}_{L \in \mathbb{R}_{>0}}$ is given by [4]:

$$
\begin{aligned}
& \mathcal{R}: \mathcal{B}_{L} \times \mathcal{B}_{M} \rightarrow \mathcal{B}_{M} \times \mathcal{B}_{L}, \quad \mathcal{R}(l, m)=\left(l^{\prime}, m^{\prime}\right), \\
& l_{i}^{\prime}:=m_{i} \frac{P_{i}(l, m)}{P_{i-1}(l, m)}, \quad m_{i}^{\prime}:=l_{i} \frac{P_{i-1}(l, m)}{P_{i}(l, m)}, \quad \text { where } \quad P_{i}(l, m):=\sum_{k=1}^{n+1} \prod_{j=k}^{n+1} l_{i+j} \prod_{j=1}^{k} m_{i+j} .
\end{aligned}
$$

Remark. In the case $\mathfrak{g}=A_{n}^{(1)}$, we have $\mathcal{B}_{L} \cong \mathcal{V}_{L}$.

\section{Epsilon systems}

\subsection{Definition of epsilon systems}

Definition 5.1. For an interval $J:=\{s, s+1, \ldots, t-1, t\} \subset I=\{1,2, \ldots, n\}$ a set of intervals $P=\left\{I_{1}, \ldots, I_{k}\right\}$ is called a partition of $J$ if disjoint intervals $I_{1}, \ldots, I_{k}$ satisfy $I_{1} \sqcup \cdots \sqcup I_{k}=J$ and $\max \left(I_{j}\right)<\min \left(I_{j+1}\right)(j=1, \ldots, k-1)$.

For a partition $P=\left\{I_{1}, \ldots, I_{k}\right\}$ of some interval $J$, set $l(P):=k$ and called the length of $P$. Let $\mathcal{J}$ be the set of all intervals in $I$. For an interval $J \in \mathcal{J}$, define

$$
\mathcal{P}_{J}:=\{P \mid P \text { is a partition of } J\} .
$$

For a partition $P=\left\{I_{1}, \ldots, I_{k}\right\}$ and symbols $\varepsilon_{I_{j}}(j=1, \ldots, k)$, define

$$
\varepsilon_{P}:=\varepsilon_{I_{1}} \cdot \varepsilon_{I_{2}} \cdots \varepsilon_{I_{k}}
$$

Definition 5.2. For an $A_{n}$-geometric crystal $\mathbb{X}=\left(X,\left\{e_{i}^{c}\right\},\left\{\gamma_{i}\right\},\left\{\varepsilon_{i}\right\}\right)$, the set of rational functions on $X$, say $E=\left\{\varepsilon_{J}, \varepsilon_{J}^{*} \mid J \in \mathcal{J}\right.$ is an interval $\}$, is called an epsilon system of $\mathbb{X}$ if they satisfy the following:

$$
\begin{aligned}
& \varepsilon_{J}\left(e_{i}^{c} x\right)= \begin{cases}c^{-1} \varepsilon_{J}(x) & \text { if } i=s, \\
\varepsilon_{J}(x) & \text { if } i \neq s-1, s, t+1,\end{cases} \\
& \varepsilon_{J}^{*}\left(e_{i}^{c} x\right)= \begin{cases}c^{-1} \varepsilon_{J}^{*}(x) & \text { if } i=t, \\
\varepsilon_{J}^{*}(x) & \text { if } i \neq s-1, t, t+1,\end{cases} \\
& \varepsilon_{J}^{*}=\sum_{P \in \mathcal{P}_{J}}(-1)^{|J|-l(P)} \varepsilon_{P}, \quad \text { for any interval } J=\{s, s+1, \ldots, t\} \subset I,
\end{aligned}
$$

and we set $\varepsilon_{J}:=\varepsilon_{i}$ for $J=\{i\}$, which is originally equipped with $\mathbb{X}$. Note that $\varepsilon_{i}^{*}=\varepsilon_{i}$. We call a geometric crystal with an epsilon system an $\varepsilon$-geometric crystal.

The actions of $e_{s-1}^{c}$ and $e_{t+1}^{c}$ will be described explicitly below, which are derived from (5.1) and (5.2).

Proposition 5.3. The above definition is well-defined, that is, for any $J \in \mathcal{J}$ we have

$$
\begin{aligned}
& \varepsilon_{J}\left(e_{i}^{c_{1}} e_{j}^{c_{2}} x\right)=\varepsilon_{J}\left(e_{j}^{c_{2}} e_{i}^{c_{1}} x\right), \quad \varepsilon_{J}^{*}\left(e_{i}^{c_{1}} e_{j}^{c_{2}} x\right)=\varepsilon_{J}^{*}\left(e_{j}^{c_{2}} e_{i}^{c_{1}} x\right) \quad \text { if } \quad a_{i j}=a_{j i}=0, \\
& \varepsilon_{J}\left(e_{i}^{c_{1}} e_{j}^{c_{1} c_{2}} e_{i}^{c_{2}} x\right)=\varepsilon_{J}\left(e_{j}^{c_{2}} e_{i}^{c_{1} c_{2}} e_{j}^{c_{1}} x\right), \\
& \varepsilon_{J}^{*}\left(e_{i}^{c_{1}} e_{j}^{c_{1} c_{2}} e_{i}^{c_{2}} x\right)=\varepsilon_{J}^{*}\left(e_{j}^{c_{2}} e_{i}^{c_{1} c_{2}} e_{j}^{c_{1}} x\right), \quad \text { if } \quad a_{i j}=a_{j i}=-1 .
\end{aligned}
$$

More precisely, we claim that if we calculate the both sides of the above equations by using (5.1) and (5.2), they coincide with each other. 
The proof will be given in the next subsection.

\section{Example 5.4.}

(i) $I=\{1,2\}: E=\left\{\varepsilon_{1}, \varepsilon_{2}, \varepsilon_{12}, \varepsilon_{21}=\varepsilon_{12}^{*}\right\}$ with the relation $\varepsilon_{21}=\varepsilon_{1} \varepsilon_{2}-\varepsilon_{12}$.

(ii) $I=\{1,2,3\}: E=\left\{\varepsilon_{1}, \varepsilon_{2}, \varepsilon_{3}, \varepsilon_{12}, \varepsilon_{12}^{*}, \varepsilon_{23}, \varepsilon_{23}^{*}, \varepsilon_{123}, \varepsilon_{123}^{*}\right\}$ with the relations for rank 2 and $\varepsilon_{123}^{*}=\varepsilon_{123}-\varepsilon_{1} \varepsilon_{23}-\varepsilon_{12} \varepsilon_{3}+\varepsilon_{1} \varepsilon_{2} \varepsilon_{3}$.

The following lemma will be needed in the sequel. Set $[l, m]:=\{l, l+1, \ldots, m-1, m\}(l \leq m)$ and if $l>m,[l, m]:=\varnothing$. We also put $\varepsilon_{\varnothing}(x)=1$. Let $\left\{\varepsilon_{J}\right\}_{J \in \mathcal{J}}$ be an epsilon system on an $\varepsilon$-geometric crystal $\mathbb{X}$.

Lemma 5.5. One of the following relations (5.5) and (5.6) is equivalent to (5.2):

$$
\begin{aligned}
& \sum_{j=s-1}^{t}(-1)^{j} \varepsilon_{[s, j]} \varepsilon_{[j+1, t]}^{*}=0 \\
& \sum_{j=s-1}^{t}(-1)^{j} \varepsilon_{[s, j]}^{*} \varepsilon_{[j+1, t]}=0
\end{aligned}
$$

for any $s, t \in I$ such that $s \leq t$.

Proof. The proof is easily done by using the induction on $t-s$.

The following describes the explicit action of $e_{i}^{c}$ on epsilon systems which is not given in Definition 5.2.

Proposition 5.6. We have the following formula for $s \leq t$ :

$$
\begin{aligned}
& \varepsilon_{[s, t]}\left(e_{t+1}^{c}(x)\right)=\varepsilon_{[s, t]}(x)+\frac{(c-1) \varepsilon_{[s, t+1]}(x)}{\varepsilon_{t+1}(x)}, \\
& \varepsilon_{[s, t]}\left(e_{s-1}^{c}(x)\right)=c \varepsilon_{[s, t]}(x)+\frac{(1-c) \varepsilon_{[s-1, t]}(x)}{\varepsilon_{s-1}(x)}, \\
& \varepsilon_{[s, t]}^{*}\left(e_{t+1}^{c}(x)\right)=c \varepsilon_{[s, t]}^{*}(x)+\frac{(1-c) \varepsilon_{[s, t+1]}^{*}(x)}{\varepsilon_{t+1}(x)}, \\
& \varepsilon_{[s, t]}^{*}\left(e_{s-1}^{c}(x)\right)=\varepsilon_{[s, t]}^{*}(x)+\frac{(c-1) \varepsilon_{[s-1, t]}^{*}(x)}{\varepsilon_{s-1}(x)} .
\end{aligned}
$$

Proof. Let us only show (5.7) since the others are shown similarly. It follows from (5.5) for $[s, t+1]$ that

$$
\varepsilon_{[s, t]}(x)=\frac{1}{\varepsilon_{t+1}(x)}\left(\varepsilon_{[s, t+1]}(x)+\sum_{i=s-1}^{t-1}(-1)^{t-i-1} \varepsilon_{[s, i]}(x) \varepsilon_{[i+1, t+1]}^{*}(x)\right) .
$$

Then applying $e_{t}^{c}$ to $x$, we get

$$
\begin{aligned}
\varepsilon_{[s, t]}\left(e_{t+1}^{c} x\right) & =\frac{1}{c^{-1} \varepsilon_{t+1}(x)}\left(\varepsilon_{[s, t+1]}(x)+c^{-1} \sum_{i=s-1}^{t-1}(-1)^{t-i-1} \varepsilon_{[s, i]}(x) \varepsilon_{[i+1, t+1]}^{*}(x)\right) \\
& =\frac{1}{\varepsilon_{t+1}(x)}\left(c \varepsilon_{[s, t+1]}(x)+\varepsilon_{[s, t]}(x) \varepsilon_{t+1}(x)-\varepsilon_{[s, t+1]}(x)\right) \\
& =\varepsilon_{[s, t]}(x)+\frac{(c-1) \varepsilon_{[s, t+1]}(x)}{\varepsilon_{t+1}(x)} .
\end{aligned}
$$

By this result, we know that all explicit forms of the action by $e_{i}^{c}$ on epsilon systems. 


\subsection{The proof of Proposition 5.3}

Let us prove Proposition 5.3. It is trivial to show (5.3). As for (5.4), the crucial cases are: for $J=[s, t]$

$$
\begin{aligned}
& \varepsilon_{J}\left(e_{s-1}^{c_{1}} e_{s}^{c_{1} c_{2}} e_{s-1}^{c_{2}} x\right)=\varepsilon_{J}\left(e_{s}^{c_{2}} e_{s-1}^{c_{1} c_{2}} e_{s}^{c_{1}} x\right), \\
& \varepsilon_{J}\left(e_{t}^{c_{1}} e_{t+1}^{c_{1} c_{2}} e_{t}^{c_{2}} x\right)=\varepsilon_{J}\left(e_{t+1}^{c_{2}} e_{t}^{c_{1} c_{2}} e_{t+1}^{c_{1}} x\right), \\
& \varepsilon_{J}^{*}\left(e_{s-1}^{c_{1}} e_{s}^{c_{1} c_{2}} e_{s-1}^{c_{2}} x\right)=\varepsilon_{J}^{*}\left(e_{s}^{c_{2}} e_{s-1}^{c_{1} c_{2}} e_{s}^{c_{1}} x\right), \\
& \varepsilon_{J}^{*}\left(e_{t}^{c_{1}} e_{t+1}^{c_{1} c_{2}} e_{t}^{c_{2}} x\right)=\varepsilon_{J}^{*}\left(e_{t+1}^{c_{2}} e_{t}^{c_{1} c_{2}} e_{t+1}^{c_{1}} x\right) .
\end{aligned}
$$

Using the results in Proposition 5.6, let us show (5.11)

$$
\begin{aligned}
& \varepsilon_{[s, t]}\left(e_{s-1}^{c_{1}} e_{s}^{c_{1} c_{2}} e_{s-1}^{c_{2}} x\right)=c_{1} \varepsilon_{[s, t]}\left(e_{s}^{c_{1} c_{2}} e_{s-1}^{c_{2}} x\right)+\frac{\left(1-c_{1}\right) \varepsilon_{[s-1, t]}\left(e_{s}^{c_{1} c_{2}} e_{s-1}^{c_{2}} x\right)}{\varepsilon_{s-1}\left(e_{s}^{c_{s} c_{2}} e_{s-1}^{c_{2}} x\right)} \\
& =c_{2}^{-1}\left(c_{2} \varepsilon_{[s, t]}(x)+\frac{\left(1-c_{2}\right) \varepsilon_{[s-1, t]}(x)}{\varepsilon_{s-1}(x)}\right)+\frac{\left(1-c_{1}\right) \varepsilon_{[s-1, t]}(x)\left(\varepsilon_{[s-1, s]}(x)+c_{2} \varepsilon_{[s-1, s]}^{*}(x)\right)}{c_{2} \varepsilon_{s-1}(x)\left(c_{1} \varepsilon_{[s-1, s]}(x)+\varepsilon_{[s-1, s]}^{*}(x)\right)} \\
& =\varepsilon_{[s, t]}(x)+\frac{\varepsilon_{[s-1, t]}(x)}{c_{2} \varepsilon_{s-1}(x)}\left(1-c_{2}+\frac{\left(1-c_{1}\right)\left(\varepsilon_{[s-1, s]}(x)+c_{2} \varepsilon_{[s-1, s]}^{*}(x)\right)}{c_{1} \varepsilon_{[s-1, s]}(x)+\varepsilon_{[s-1, s]}^{*}(x)}\right) \\
& =\varepsilon_{[s, t]}(x)+\frac{\varepsilon_{[s-1, t]}(x)}{c_{2} \varepsilon_{s-1}(x)} \frac{\left(1-c_{1} c_{2}\right)\left(\varepsilon_{[s-1, s]}(x)+\varepsilon_{[s-1, s]}^{*}(x)\right)}{\left.c_{1} \varepsilon_{[s-1, s]}(x)+\varepsilon_{[s-1, s]}^{*}(x)\right)} \\
& =\varepsilon_{[s, t]}(x)+\frac{\left(1-c_{1} c_{2}\right) \varepsilon_{[s-1, t]}(x) \varepsilon_{s}(x)}{c_{2}\left(c_{1} \varepsilon_{[s-1, s]}(x)+\varepsilon_{[s-1, s]}^{*}(x)\right)}
\end{aligned}
$$

where the last equality is derived from $\varepsilon_{[s-1, s]}(x)+\varepsilon_{[s-1, s]}^{*}(x)=\varepsilon_{s-1}(x) \varepsilon_{s}(x)$. We also have

$$
\begin{aligned}
\varepsilon_{[s, t]}\left(e_{s}^{c_{2}} e_{s-1}^{c_{1} c_{2}} e_{s}^{c_{1}} x\right) & =c_{2}^{-1} \varepsilon_{[s, t]}\left(e_{s-1}^{c_{1} c_{2}} e_{s}^{c_{1}} x\right)=c_{2}^{-1}\left(c_{1} c_{2} \varepsilon_{[s, t]}\left(e_{s}^{c_{1}} x\right)+\frac{\left(1-c_{1} c_{2}\right) \varepsilon_{[s-1, t]}\left(e_{s}^{c_{1}} x\right)}{\varepsilon_{s-1}\left(e_{s}^{c_{1}} x\right)}\right) \\
& =\varepsilon_{[s, t]}(x)+\frac{\left(1-c_{1} c_{2}\right) \varepsilon_{[s-1, t]}(x) \varepsilon_{s}(x)}{c_{2}\left(c_{1} \varepsilon_{[s-1, s]}(x)+\varepsilon_{[s-1, s]}^{*}(x)\right)} .
\end{aligned}
$$

Thus, we obtained (5.11). The others are also shown by direct calculations:

$$
\begin{aligned}
& \varepsilon_{[s, t]}\left(e_{t}^{c_{1}} e_{t+1}^{c_{1} c_{2}} e_{t}^{c_{2}} x\right)=\varepsilon_{[s, t]}(x)+\frac{\left(c_{1} c_{2}-1\right) \varepsilon_{[s, t+1]}(x) \varepsilon_{t}(x)}{\varepsilon_{[t, t+1]}(x)+c_{2} \varepsilon_{[t, t+1]}^{*}(x)}=\varepsilon_{[s, t]}\left(e_{t+1}^{c_{2}} e_{t}^{c_{1} c_{2}} e_{t+1}^{c_{1}} x\right), \\
& \varepsilon_{[s, t]}^{*}\left(e_{s-1}^{c_{1}} e_{s}^{c_{1} c_{2}} e_{s-1}^{c_{2}} x\right)=\varepsilon_{[s, t]}^{*}(x)+\frac{\left(c_{1} c_{2}-1\right) \varepsilon_{[s-1, t]}^{*}(x) \varepsilon_{s}(x)}{c_{1} \varepsilon_{[s-1, s]}(x)+\varepsilon_{[s-1, s]}^{*}(x)}=\varepsilon_{[s, t]}^{*}\left(e_{s}^{c_{2}} e_{s-1}^{c_{1} c_{2}} e_{s}^{c_{1}} x\right), \\
& \varepsilon_{[s, t]}^{*}\left(e_{t}^{c_{1}} e_{t+1}^{c_{1} c_{2}} e_{t}^{c_{2}} x\right)=\varepsilon_{[s, t]}^{*}(x)+\frac{\left(1-c_{1} c_{2}\right) \varepsilon_{[s, t+1]}^{*}(x) \varepsilon_{t}(x)}{c_{1}\left(\varepsilon_{[t, t+1]}(x)+c_{2} \varepsilon_{[t, t+1]}^{*}(x)\right)}=\varepsilon_{[s, t]}^{*}\left(e_{t+1}^{c_{2}} e_{t}^{c_{1} c_{2}} e_{t+1}^{c_{1}} x\right) .
\end{aligned}
$$

Let $\mathfrak{g}$ be a Kac-Moody Lie algebra associated with the index set $I$ and $\mathfrak{g}_{J}$ be a subalgebra associated with a subset $J \subset I$. Let $\mathbf{X}=\left(X,\left\{\gamma_{i}\right\},\left\{\varepsilon_{i}\right\},\left\{e_{i}\right\}\right)_{i \in I}$ be a g-geometric crystal. Then it has naturally a $\mathfrak{g}_{J}$-geometric crystal structure and denote it by $\mathbf{X}_{J}$.

Definition 5.7. In the above setting, if $\mathfrak{g}_{J}$ is isomorphic to the Lie algebra of type $A_{n}$ for some $n$ and the geometric crystal $\mathbf{X}_{J}$ has an epsilon system $E_{\mathbf{X}_{J}}$ of type $A_{n}$, then we call it a local epsilon system of type $A_{n}$ associated with the index set $J$.

Remark. An $\varepsilon$-geometric crystal has naturally a local epsilon system associated with each sub-interval of $I$.

Example 5.8. In Example 5.4(ii), $\left\{\varepsilon_{2}, \varepsilon_{3}, \varepsilon_{23}, \varepsilon_{23}^{*}\right\} \subset E$ is a local epsilon system of type $A_{2}$ associated with the interval $\{2,3\}$. 


\subsection{Product structures on epsilon systems}

Theorem 5.9. Let $\mathbb{X}$ and $\mathbb{Y}$ be $\varepsilon$-geometric crystals. Suppose that the product $\mathbb{X} \times \mathbb{Y}$ has a geometric crystal structure. Then $\mathbb{X} \times \mathbb{Y}$ turns out to be an $\varepsilon$-geometric crystal as follows: for $\varepsilon$-systems $E_{\mathbb{X}}:=\left\{\varepsilon_{J}^{X}, \varepsilon_{J}^{X *}\right\}_{J \in \mathcal{J}}$ of $\mathbb{X}$ and $E_{\mathbb{Y}}:=\left\{\varepsilon_{J}^{Y}, \varepsilon_{J}^{Y *}\right\}_{J \in \mathcal{J}}$ of $\mathbb{Y}$, set

$$
\begin{aligned}
& \varepsilon_{[s, t]}(x, y):=\sum_{k=s-1}^{t} \frac{\varepsilon_{[s, k]}^{Y}(y) \varepsilon_{[k+1, t]}^{X}(x)}{\prod_{j=s}^{k} \gamma_{j}^{X}(x)}, \\
& \varepsilon_{[s, t]}^{*}(x, y):=\sum_{k=s-1}^{t} \frac{\varepsilon_{[s, k]}^{* X}(x) \varepsilon_{[k+1, t]}^{* Y}(y)}{\prod_{j=k+1}^{t} \gamma_{j}^{X}(x)} .
\end{aligned}
$$

Then $E_{\mathbb{X} \times \mathbb{Y}}:=\left\{\varepsilon_{[s, t]}(x, y), \varepsilon_{[s, t]}^{*}(x, y)\right\}_{[s, t] \in \mathcal{J}}$ defines an epsilon system of $\mathbb{X} \times \mathbb{Y}$.

Example 5.10. We have

$$
\varepsilon_{123}(x, y)=\varepsilon_{123}(x)+\frac{\varepsilon_{1}(y) \varepsilon_{23}(x)}{\gamma_{1}(x)}+\frac{\varepsilon_{12}(y) \varepsilon_{3}(x)}{\gamma_{1}(x) \gamma_{2}(x)}+\frac{\varepsilon_{123}(y)}{\gamma_{1}(x) \gamma_{2}(x) \gamma_{3}(x)}
$$

Proof. First, we shall show (5.1). For $c \in \mathbb{C}^{\times}$and $(x, y) \in X \times Y$, set $e_{i}^{c}(x, y)=\left(e_{i}^{c_{1}} x, e_{i}^{c_{2}} y\right)$ where

$$
c_{1}:=\frac{c \varphi_{i}(x)+\varepsilon_{i}(y)}{\varphi_{i}(x)+\varepsilon_{i}(y)}, \quad c_{2}:=\frac{c}{c_{1}}, \quad \varphi_{i}(x):=\varepsilon_{i}(x) \gamma_{i}(x) .
$$

Let us see $\varepsilon_{[s, t]}\left(e_{s}^{c}(x, y)\right)=c^{-1} \varepsilon_{[s, t]}(x, y)$. Each summand $\frac{\varepsilon_{[s, k]}^{Y}(y) \varepsilon_{[k+1, t]}^{X}(x)}{\prod_{j=s}^{k} \gamma_{j}^{X}(x)}$ in (5.15) is changed by the action of $e_{s}^{c}$ as follows (we omit the superscripts $\mathbb{X}$ and $\mathbb{Y}$ ):

$$
\begin{aligned}
& \varepsilon_{[s, t]}\left(e_{s}^{c_{1}} x\right)=c_{1}^{-1} \varepsilon_{[s, t]}(x), \quad k=s-1, \\
& \frac{\varepsilon_{s}\left(e_{s}^{c_{2}} y\right) \varepsilon_{[s+1, t]}\left(e_{s}^{c_{1}} x\right)}{\gamma_{s}\left(e_{s}^{c_{1}} x\right)}=\frac{\varepsilon_{s}(y)}{c_{1}^{2} c_{2} \gamma_{s}(x)}\left(c_{1} \varepsilon_{[s+1, t]}(x)+\frac{\left(1-c_{1}\right) \varepsilon_{[s, t]}(x)}{\varepsilon_{s(x)}}\right), \quad k=s, \\
& \frac{\varepsilon_{[s, k]}\left(e_{s}^{c_{2}} y\right) \varepsilon_{[k+1, t]}\left(e_{s}^{c_{1}} x\right)}{\gamma_{s}\left(e_{s}^{c_{1}} x\right) \cdots \gamma_{k}\left(e_{s}^{c_{1}} x\right)}=\frac{\varepsilon_{[s, k]}(y) \varepsilon_{[k+1, t]}(x)}{c \gamma_{s}(x) \cdots \gamma_{k}(x)}, \quad k>s,
\end{aligned}
$$

where the second formula is obtained by (5.8). Now, taking the summation of (5.17) and (5.18), we have

$$
\begin{aligned}
\varepsilon_{[s, t]}(x) & \left(c_{1}^{-1}+\frac{\left(1-c_{1}\right) \varepsilon_{s}(y)}{c c_{1} \varphi_{s}(x)}\right)+\frac{\varepsilon_{s}(y) \varepsilon_{[s+1, t]}(x)}{c \gamma_{s}(x)} \\
= & c_{1}^{-1} \varepsilon_{[s, t]}(x) \frac{c \varphi_{s}(x)+\varepsilon_{s}(y)}{c\left(\varphi_{s}(x)+\varepsilon_{s}(y)\right)}+\frac{\varepsilon_{s}(y) \varepsilon_{[s+1, t]}(x)}{c \gamma_{s}(x)}=c^{-1}\left(\varepsilon_{[s, t]}(x)+\frac{\varepsilon_{s}(y) \varepsilon_{[s+1, t]}(x)}{\gamma_{s}(x)}\right) .
\end{aligned}
$$

Thus we have $\varepsilon_{[s, t]}\left(e_{s}^{c}(x, y)\right)=c^{-1} \varepsilon_{[s, t]}(x)$. The others are obtained by the similar argument.

Next, let us show (5.2). Set the right hand-side of (5.15) (resp. (5.16)) $X_{[s, t]}(x, y)$ (resp. $\left.X_{[s, t]}^{*}(x, y)\right)$. By Lemma 5.5, it suffices to show that the relation (5.5) holds for $X_{[s, t]}(x, y)$ and $X_{[s, t]}^{*}(x, y)$

$$
\sum_{j=s-1}^{t}(-1)^{j} X_{[s, j]}(x, y) X_{[j+1, t]}^{*}(x, y)
$$




$$
\begin{aligned}
& =\sum_{j=s-1}^{t}(-1)^{j}\left(\sum_{k=s-1}^{j} \frac{\varepsilon_{[s, k]}(y) \varepsilon_{[k+1, j]}(x)}{\prod_{p=s}^{k} \gamma_{p}(x)}\right)\left(\sum_{m=j}^{t} \frac{\varepsilon_{[j+1, m]}^{*}(x) \varepsilon_{[m+1, t]}^{*}(y)}{\prod_{q=m+1}^{t} \gamma_{q}(x)}\right) \\
& =\sum_{s-1 \leq k \leq m \leq t} \frac{\varepsilon_{[s, k]}(y)}{\prod_{p=s}^{k} \gamma_{p}(x)} \frac{\varepsilon_{[m+1, t]}^{*}(y)}{\prod_{q=m+1}^{t} \gamma_{q}(x)}\left(\sum_{j=k}^{m}(-1)^{j} \varepsilon_{[k+1, j]}(x) \varepsilon_{[j+1, m]}^{*}(x)\right) \\
& =\frac{1}{\prod_{p=s}^{t} \gamma_{p}(x)} \sum_{s-1 \leq k \leq t}(-1)^{k} \varepsilon_{[s, k]}(y) \varepsilon_{[k+1, t]}^{*}(y)=0
\end{aligned}
$$

since $\sum_{j=k}^{m}(-1)^{j} \varepsilon_{[k+1, j]}(x) \varepsilon_{[j+1, m]}^{*}(x)=0$ for $k<m$ and $\sum_{s-1 \leq k \leq t}(-1)^{k} \varepsilon_{[s, k]}(y) \varepsilon_{[k+1, t]}^{*}(y)=0$ by (5.5). Hence, we showed that $\left\{X_{[s, t]}(x, y), X_{[s, t]}^{*}(x, y) \mid s, t \in I(s \leq t)\right\}$ satisfy (5.5), which is equivalent to (5.2) by Lemma 5.5.

Example 5.11. $D_{5}^{(1)}$-case $[3,4]$. Set the index set $I=\{0,1,2,3,4,5\}$. The geometric crystal $\mathcal{B}_{L}$ is defined as follows:

$$
\begin{aligned}
& \mathcal{B}_{L}:=\left\{l=\left(l_{1}, \ldots, l_{5}, \bar{l}_{4}, \ldots, \bar{l}_{1}\right) \in\left(\mathbb{C}^{\times}\right)^{9} \mid l_{1} l_{2} \ldots \bar{l}_{2} \bar{l}_{1}=L\right\}, \\
& \varepsilon_{0}(l)=l_{1}\left(\frac{l_{2}}{\bar{l}_{2}}+1\right), \quad \varepsilon_{i}(l)=\bar{l}_{i}\left(\frac{l_{i+1}}{\bar{l}_{i+1}}+1\right) \quad(i=1,2,3), \quad \varepsilon_{4}(l)=l_{5} \bar{l}_{4}, \quad \varepsilon_{5}(l)=\bar{l}_{4}, \\
& \gamma_{0}(l)=\frac{\bar{l}_{1} \bar{l}_{2}}{l_{1} l_{2}}, \quad \gamma_{i}(l)=\frac{l_{i} \bar{l}_{i+1}}{\bar{l}_{i} l_{i+1}} \quad(i=1,2,3), \quad \gamma_{4}(l)=\frac{l_{4}}{l_{5} \bar{l}_{4}}, \quad \gamma_{5}(l)=\frac{l_{4} l_{5}}{\bar{l}_{4}}, \\
& e_{0}^{c}(l)=\left(\xi_{1}^{-1} l_{1}, c^{-1} \xi_{1} l_{2}, \ldots, \xi_{1} \bar{l}_{2}, c \xi_{1}^{-1} \bar{l}_{1}\right), \\
& e_{i}^{c}(l)=\left(\ldots, c \xi_{i}^{-1} l_{i}, c^{-1} \xi_{i} l_{i+1}, \ldots, \xi_{i} \bar{l}_{i+1}, \xi_{i}^{-1} \bar{l}_{i}, \ldots\right) \quad(i=1,2,3), \\
& e_{4}^{c}(l)=\left(\ldots, c \cdot l_{4}, c^{-1} l_{5}, \ldots\right), \quad e_{5}^{c}(l)=\left(\ldots, c \cdot l_{5}, c^{-1} \bar{l}_{4}, \ldots\right) .
\end{aligned}
$$

where $\xi_{i}=\frac{l_{i+1}+c \bar{l}_{i+1}}{l_{i+1}+\bar{l}_{i+1}}$. There are several local epsilon systems of type $A_{4}$ in the $D_{5}^{(1)}$-geometric crystal $\mathcal{B}_{L}$ associated with e.g., $\{0,2,3,4\},\{0,2,3,5\} \subset I$. Then, we have

$$
\begin{aligned}
& \varepsilon_{02}(l)=l_{1} l_{2}\left(\frac{l_{3}}{\bar{l}_{3}}+1\right), \quad \varepsilon_{02}^{*}(l)=l_{1} \bar{l}_{2}\left(\frac{l_{3}}{\bar{l}_{3}}+1\right), \quad \varepsilon_{i i+1}(l)=\bar{l}_{i} l_{i+1}\left(\frac{l_{i+2}}{\bar{l}_{i+2}}+1\right), \\
& \varepsilon_{i i+1}^{*}(l)=\bar{l}_{i} \bar{l}_{i+1}\left(\frac{l_{i+2}}{\bar{l}_{i+2}}+1\right) \quad(i=1,2), \quad \varepsilon_{34}(l)=\bar{l}_{3} l_{4} l_{5}, \\
& \varepsilon_{34}^{*}(l)=\bar{l}_{3} \bar{l}_{4} l_{5}, \varepsilon_{35}(l)=\bar{l}_{3} l_{4}, \quad \varepsilon_{35}^{*}(l)=\bar{l}_{3} \bar{l}_{4}, \\
& \varepsilon_{023}(l)=l_{1} l_{2} l_{3}\left(\frac{l_{4}}{\bar{l}_{4}}+1\right), \quad \varepsilon_{023}^{*}(l)=l_{1} \bar{l}_{2} \bar{l}_{3}\left(\frac{l_{4}}{\bar{l}_{4}}+1\right), \\
& \varepsilon_{234}(l)=\bar{l}_{2} l_{3} l_{4} l_{5}, \quad \varepsilon_{234}^{*}(l)=\bar{l}_{2} \bar{l}_{3} \bar{l}_{4} l_{5}, \\
& \varepsilon_{0234}(l)=l_{1} l_{2} l_{3} l_{4} l_{5}, \quad \varepsilon_{0234}^{*}(l)=l_{1} \bar{l}_{2} \bar{l}_{3} \bar{l}_{4} l_{5}, \quad l_{0}^{\circ} \\
& \varepsilon_{0235}(l)=l_{1} l_{2} l_{3} l_{4}, \quad \varepsilon_{0235}^{*}(l)=l_{1} \bar{l}_{2} \bar{l}_{3} \bar{l}_{4}, \quad \ldots \quad \text { etc. }
\end{aligned}
$$

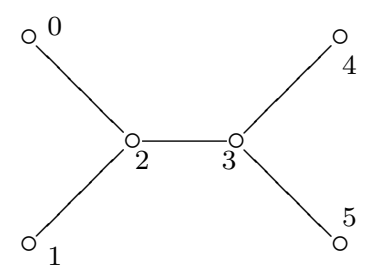

\section{Epsilon systems on the Borel subgroup $B^{-}$}

We shall show that there exists a canonical epsilon system on the (opposite) Borel subgroup $B^{-}$. Indeed, it is a prototype of general epsilon systems and derives an epsilon system to any $A_{n^{-}}$ geometric crystals induced from unipotent crystals. 
In this section we shall identify $B^{-}$with the set of lower triangular matrices in $S L_{n+1}(\mathbb{C})$. Let $T:=\left\{\operatorname{diag}\left(t_{1}, \ldots, t_{n+1}\right) \in S L_{n+1}(\mathbb{C})\right\}$ be the maximal torus in $B^{-}$. For $x \in B^{-}$there exist a unique unipotent matrix $x_{-} \in U^{-}$and a unique diagonal matrix $x_{0} \in T$ such that $x=x_{-} x_{0}$. Then the geometric crystal structure on $B^{-}$is described as follows: For $x \in B^{-}$, write $x_{0}=\operatorname{diag}\left(t_{1}, \ldots, t_{n+1}\right)$ and $(i, j)$-entry of $x_{-}$as

$$
\left(x_{-}\right)_{i, j}= \begin{cases}u_{j} & \text { if } i=j+1 \\ u_{j, i-1} & \text { if } i>j+1 \\ 1 & \text { if } i=j \\ 0 & \text { otherwise. }\end{cases}
$$

For example, for $n=2$-case,

$$
x_{-}=\left(\begin{array}{ccc}
1 & 0 & 0 \\
u_{1} & 1 & 0 \\
u_{12} & u_{2} & 1
\end{array}\right), \quad x_{0}=\left(\begin{array}{ccc}
t_{1} & 0 & 0 \\
0 & t_{2} & 0 \\
0 & 0 & t_{3}
\end{array}\right), \quad x=\left(\begin{array}{ccc}
t_{1} & 0 & 0 \\
t_{1} u_{1} & t_{2} & 0 \\
t_{1} u_{12} & t_{2} u_{2} & t_{3}
\end{array}\right) .
$$

Now, the rational functions $\gamma_{i}$ and $\varepsilon_{i}$ are given by $\gamma_{i}(x)=t_{i} / t_{i+1}$ and $\varepsilon_{i}(x)=u_{i}$. The action $e_{i}^{c}$ $\left(c \in \mathbb{C}^{\times}\right)$is given by

$$
e_{i}^{c}(x)=x_{i}\left(\frac{c-1}{\varepsilon_{i}(x)}\right)(x)=x_{i}\left(\frac{c-1}{\varepsilon_{i}(x)}\right) \cdot x \cdot x_{i}\left(\frac{c^{-1}-1}{\varphi_{i}(x)}\right),
$$

where $x_{i}(z)=\operatorname{Id}_{n+1}+z E_{i, i+1} \in B \subset S L_{n+1}(\mathbb{C})$.

Proposition 6.1. In the above setting, the epsilon system on $B^{-} \subset S L_{n+1}(\mathbb{C})$ are given by:

$$
\varepsilon_{[s, t]}(x)=u_{s, t}, \quad \varepsilon_{[s, t]}^{*}(x)=\operatorname{det}\left(M_{s, t}\right), \quad s<t,
$$

where $M_{s, t}$ is the minor of $x_{-}$as:

$$
M_{s, t}:=\left(\begin{array}{ccccc}
u_{s} & 1 & 0 & \cdots & 0 \\
u_{s, s+1} & u_{s+1} & 1 & \cdots & 0 \\
& \cdots & \cdots & \cdots & \cdots \\
u_{s, t-1} & u_{s+1, t-1} & \cdots & \cdots & 1 \\
u_{s, t} & u_{s+1, t} & \cdots & u_{t-1, t} & u_{t}
\end{array}\right) .
$$

Proof. By direct calculations, for $x \in B^{-}$we have

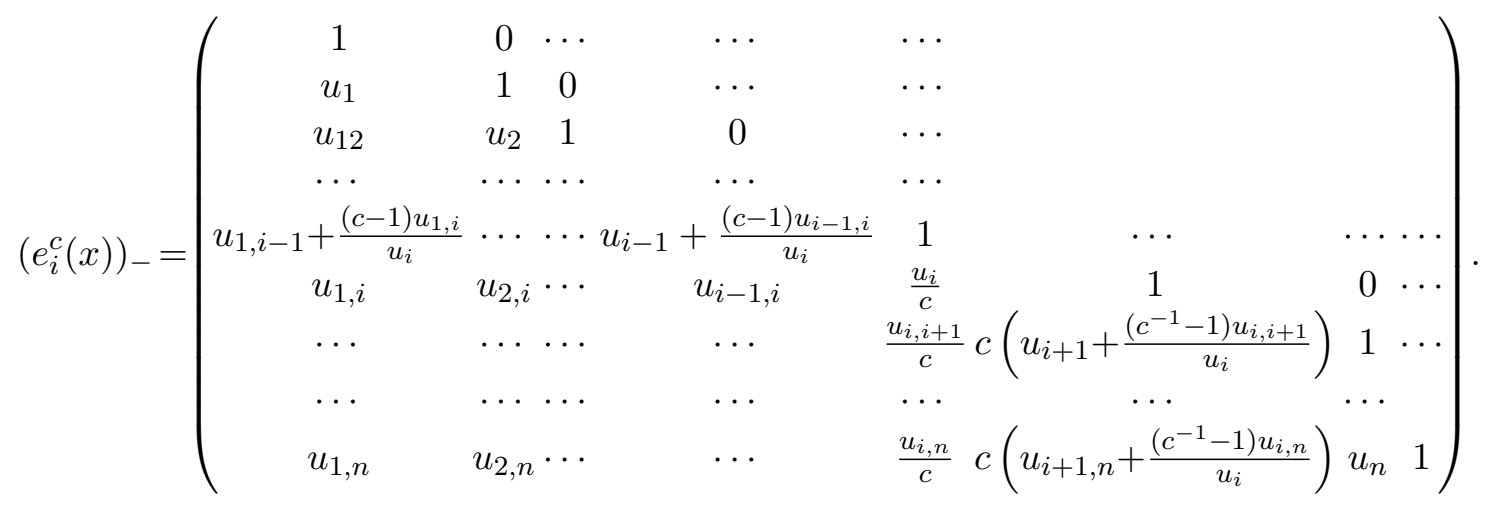

The formula (5.1) in Definition 5.2 is shown by using this.

We can easily see

$$
\operatorname{det}\left(M_{s, t}\right)=u_{s} \operatorname{det}\left(M_{s+1, t}\right)-u_{s, s+1} \operatorname{det}\left(M_{s+2, t}\right)+\cdots+(-1)^{t-s} u_{s, t} .
$$

Thus, by the induction on $t-s$, we obtain (5.2). 
Next, we shall see an epsilon system on a geometric crystal induced from a unipotent crystal. Let $(X, f)$ be a unipotent $S L_{n+1}(\mathbb{C})$ crystal, where $f: X \rightarrow B^{-}$is a $U$-morphism. We assume that any rational function $\varepsilon_{i}$ is not identically zero. By Theorem 2.5, we get the geometric crystal $\mathbb{X}=\left(X,\left\{e_{i}^{c}\right\},\left\{\gamma_{i}\right\},\left\{\varepsilon_{i}\right\}\right)$.

Theorem 6.2. The geometric crystal $\mathbb{X}$ as above is an $\varepsilon$-geometric crystal. Indeed, by setting

$$
\begin{aligned}
& \varepsilon_{[s, t]}^{\mathbb{X}}(x):=\varepsilon_{[s, t]}^{B^{-}}(f(x)), \\
& \varepsilon_{[s, t]}^{* \mathbb{X}}(x)=\sum_{P \in \mathcal{P}_{[s, t]}}(-1)^{(t-s+1)-l(P)} \varepsilon_{P}^{\mathbb{X}}(x),
\end{aligned}
$$

the set $E_{\mathbb{X}}:=\left\{\varepsilon_{[s, t]}^{\mathbb{X}}(x), \varepsilon_{[s, t]}^{* \mathbb{X}}(x) \mid 1 \leq s \leq t \leq n\right\}$ defines an epsilon system on $\mathbb{X}$.

Proof. The relation (5.2) is immediate from (6.2). Since $f\left(e_{i}^{c}(x)\right)=e_{i}^{c}(f(x))$ and $\varepsilon_{[s, t]}^{B^{-}}$satisfies (5.1), we can easily obtain (5.1) for $\varepsilon_{[s, t]}^{\mathbb{X}}$.

\section{$7 \quad$ Epsilon systems and tropical $\mathrm{R}$ maps}

Let $\mathbb{X}=\left(X,\left\{e_{i}^{c}\right\},\left\{\gamma_{i}\right\}, E_{\mathbb{X}}\right)$ and $\mathbb{Y}=\left(Y,\left\{e_{i}^{c}\right\},\left\{\gamma_{i}\right\}, E_{\mathbb{Y}}\right)$ be $\varepsilon$-geometric crystals, where $E_{\mathbb{X}}$ $\left(\right.$ resp. $\left.E_{\mathbb{Y}}\right)$ is the epsilon system of $\mathbb{X}($ resp. $\mathbb{Y})$. Suppose that there exists a tropical R-map

$\mathcal{R}: X \times Y \rightarrow Y \times X$.

Let $E_{\mathbb{X} \times \mathbb{Y}}=\left\{\varepsilon_{J}^{\mathbb{X} \times \mathbb{Y}}, \varepsilon_{J}^{* \mathbb{X} \times \mathbb{Y}}\right\}_{J \in \mathcal{J}}$ (resp. $E_{\mathbb{Y} \times \mathbb{X}}=\left\{\varepsilon_{J}^{\mathbb{Y} \times \mathbb{X}}, \varepsilon_{J}^{* \mathbb{Y} \times \mathbb{X}}\right\}_{J \in \mathcal{J}}$ ) be the epsilon system on $\mathbb{X} \times \mathbb{Y}$ (resp. $\mathbb{Y} \times \mathbb{X})$ obtained from $E_{\mathbb{X}}$ and $E_{\mathbb{Y}}$ by Theorem 5.9.

Theorem 7.1. The epsilon systems $E_{\mathbb{X} \times \mathbb{Y}}$ and $E_{\mathbb{Y} \times \mathbb{X}}$ are invariant by the action of $\mathcal{R}$ in the following sense:

$$
\varepsilon_{J}^{\mathbb{Y} \times \mathbb{X}}(\mathcal{R}(x, y))=\varepsilon_{J}^{\mathbb{X} \times \mathbb{Y}}(x, y), \quad \varepsilon_{J}^{* \mathbb{Y} \times \mathbb{X}}(\mathcal{R}(x, y))=\varepsilon_{J}^{* \mathbb{X} \times \mathbb{Y}}(x, y),
$$

for any $J \in \mathcal{J}$.

Proof. Let us show the theorem by the induction on the length of intervals $J$, denoted $|J|$. Assume that we have (7.1) for $|J|<n$. The case $|J|=1$ is obtained from (4.2) in Definition 4.1: the definition of tropical R maps. Now, we shall show

$$
\varepsilon_{I}^{\mathbb{Y} \times \mathbb{X}}(\mathcal{R}(x, y))=\varepsilon_{I}^{\mathbb{X} \times \mathbb{Y}}(x, y), \quad \varepsilon_{I}^{* \mathbb{Y} \times \mathbb{X}}(\mathcal{R}(x, y))=\varepsilon_{I}^{* \mathbb{X} \times \mathbb{Y}}(x, y) .
$$

By Theorem 5.9, we have

$$
\varepsilon_{I}^{* \mathbb{X} \times \mathbb{Y}}(x, y)=\sum_{P \in \mathcal{P}_{I}}(-1)^{n-l(P)} \varepsilon_{P}^{\mathbb{X} \times \mathbb{Y}}(x, y) .
$$

In the above summation, if a partition $P \in \mathcal{P}_{I}$ is different from $I=\{1,2, \ldots, n\}$, then $\varepsilon_{P}^{\mathbb{X} \times \mathbb{Y}}(x, y)$ is invariant by the induction hypothesis. Thus, we have

$$
\varepsilon_{I}^{* \mathbb{Y} \times \mathbb{X}}(\mathcal{R}(x, y))=(-1)^{n-1} \varepsilon_{I}^{\mathbb{Y} \times \mathbb{X}}(\mathcal{R}(x, y))+\sum_{P \in \mathcal{P}_{I}, P \neq I}(-1)^{n-l(P)} \varepsilon_{P}^{\mathbb{X} \times \mathbb{Y}}(x, y) .
$$

It follows from (7.2) and (7.3) that

$$
\varepsilon_{I}^{* \mathbb{Y} \times \mathbb{X}}(\mathcal{R}(x, y))-\varepsilon_{I}^{* \mathbb{X} \times \mathbb{Y}}(x, y)=(-1)^{n-1}\left(\varepsilon_{I}^{\mathbb{Y} \times \mathbb{X}}(\mathcal{R}(x, y))-\varepsilon_{I}^{\mathbb{X} \times \mathbb{Y}}(x, y)\right) .
$$


Here, using (5.1) we have

$$
\begin{aligned}
& \varepsilon_{I}^{* \mathbb{Y} \times \mathbb{X}}\left(\mathcal{R} e_{1}^{c}(x, y)\right)=\varepsilon_{I}^{* \mathbb{Y} \times \mathbb{X}}\left(e_{1}^{c} \mathcal{R}(x, y)\right)=\varepsilon_{I}^{* \mathbb{Y} \times \mathbb{X}}(\mathcal{R}(x, y)), \\
& \varepsilon_{I}^{* \mathbb{X} \times \mathbb{Y}}\left(e_{1}^{c}(x, y)\right)=\varepsilon_{I}^{* \mathbb{X} \times \mathbb{Y}}(x, y), \\
& \varepsilon_{I}^{\mathbb{Y} \times \mathbb{X}}\left(\mathcal{R} e_{1}^{c}(x, y)\right)=\varepsilon_{I}^{\mathbb{Y} \times \mathbb{X}}\left(e_{1}^{c} \mathcal{R}(x, y)\right)=c^{-1} \varepsilon_{I}^{\mathbb{Y} \times \mathbb{X}}(\mathcal{R}(x, y)), \\
& \varepsilon_{I}^{\mathbb{X} \times \mathbb{Y}}\left(e_{1}^{c}(x, y)\right)=c^{-1} \varepsilon_{I}^{\mathbb{X} \times \mathbb{Y}}(x, y) .
\end{aligned}
$$

Applying these to (7.4), for any $c \in \mathbb{C}^{\times}$one has

$$
\varepsilon_{I}^{* \mathbb{Y} \times \mathbb{X}}(\mathcal{R}(x, y))-\varepsilon_{I}^{* \mathbb{X} \times \mathbb{Y}}(x, y)=(-1)^{n-1} c^{-1}\left(\varepsilon_{I}^{\mathbb{Y} \times \mathbb{X}}(\mathcal{R}(x, y))-\varepsilon_{I}^{\mathbb{X} \times \mathbb{Y}}(x, y)\right) .
$$

Hence, we obtain

$$
\varepsilon_{I}^{* \mathbb{Y} \times \mathbb{X}}(\mathcal{R}(x, y))=\varepsilon_{I}^{* \mathbb{X} \times \mathbb{Y}}(x, y), \quad \varepsilon_{I}^{\mathbb{Y} \times \mathbb{X}}(\mathcal{R}(x, y))=\varepsilon_{I}^{\mathbb{X} \times \mathbb{Y}}(x, y),
$$

which completes the proof.

Observing this proof, it is easy to get the following:

Corollary 7.2. Let $\mathbb{X}$ (resp. $\mathbb{Y}$ ) be an $\varepsilon$-geometric crystal with the epsilon system $E_{\mathbb{X}}=$ $\left\{\varepsilon_{J}^{\mathbb{X}}, \varepsilon_{J}^{* \mathbb{X}}\right\}_{J \in \mathcal{J}}$ (resp. $\left.E_{\mathbb{Y}}=\left\{\varepsilon_{J}^{\mathbb{Y}}, \varepsilon_{J}^{* \mathbb{Y}}\right\}_{J \in \mathcal{J}}\right)$ and $F: \mathbb{X} \rightarrow \mathbb{Y}$ a homomorphism of geometric crystals, that is, $F$ is a rational map commuting with the action of any $e_{i}$ and preserving the functions $\varepsilon_{i}$ and $\gamma_{i}$. Then we obtain for any $J \in \mathcal{J}$

$$
\varepsilon_{J}^{\mathbb{Y}}(F(x))=\varepsilon_{J}^{\mathbb{X}}(x), \quad \varepsilon_{J}^{* \mathbb{Y}}(F(x))=\varepsilon_{J}^{* \mathbb{X}}(x) .
$$

\subsection{Application-uniqueness of $A_{n}^{(1)}$-tropical R map}

Let $\left\{\mathcal{B}_{L}\right\}_{L>0}$ be the family of $A_{n}^{(1)}$-geometric crystals as in Example 4.3. If we forget the index 0, $\mathcal{B}_{L}$ can be seen as an $A_{n}$-geometric crystal and is equipped with following local epsilon system of type $A_{n}$ :

$$
\varepsilon_{i}(l)=l_{i+1}=\varepsilon_{i}^{*}(l), \quad \varepsilon_{[s, t]}^{*}(l)=0, \quad \varepsilon_{[s, t]}(l)=l_{s+1} l_{s+2} \cdots l_{t+1},
$$

for $l=\left(l_{1}, \ldots, l_{n+1}\right) \in \mathcal{B}_{L}$.

Proposition 7.3. Let $\mathcal{R}: \mathcal{B}_{L} \times \mathcal{B}_{M} \rightarrow \mathcal{B}_{M} \times \mathcal{B}_{L}$ be the tropical $R$ map in Example 4.3. Then $\mathcal{R}$ is the unique tropical $R$ maps from $\mathcal{B}_{L} \times \mathcal{B}_{M}$ to $\mathcal{B}_{M} \times \mathcal{B}_{L}$.

Proof. For $\mathcal{B}_{L} \times \mathcal{B}_{M}$, by Theorem 5.9 we have

$$
\begin{aligned}
& \varepsilon_{i}(l, m)=l_{i+1}+\frac{l_{i+1} m_{i+1}}{l_{i}}, \quad i=1, \ldots, n, \\
& \varepsilon_{i, i+1}^{*}(l, m)=\varepsilon_{i, i+1}^{*}(l)+\frac{\varepsilon_{i+1}(m) \varepsilon_{i}(l)}{\gamma_{i+1}(l)}+\frac{\varepsilon_{i, i+1}^{*}(m)}{\gamma_{i}(l) \gamma_{i+1}(l)}=l_{i+2} m_{i+2}, \quad i=1, \ldots, n-1 .
\end{aligned}
$$

Set $\tilde{l}:=L^{\frac{1}{n+1}}, \tilde{m}:=M^{\frac{1}{n+1}}, l_{0}:=(\tilde{l}, \ldots, \tilde{l}) \in \mathcal{B}_{L}$ and $m_{0}:=(\tilde{m}, \ldots, \tilde{m}) \in \mathcal{B}_{M}$. Then it is immediate from the explicit form of $\mathcal{R}$ as in (4.5) that

$$
\mathcal{R}\left(l_{0}, m_{0}\right)=\left(m_{0}, l_{0}\right) .
$$

Let $\mathcal{R}^{\prime}$ be an arbitrary tropical R-map from $\mathcal{B}_{L} \times \mathcal{B}_{M}$ to $\mathcal{B}_{M} \times \mathcal{B}_{L}$. Set $\left(l^{\prime}, m^{\prime}\right):=\mathcal{R}^{\prime}\left(l_{0}, m_{0}\right) \in$ $\mathcal{B}_{M} \times \mathcal{B}_{L}$. By Theorem 7.1, for any interval $J$ we have

$$
\varepsilon_{J}\left(l^{\prime}, m^{\prime}\right)=\varepsilon_{J}\left(\mathcal{R}^{\prime}\left(l_{0}, m_{0}\right)\right)=\varepsilon_{J}\left(l_{0}, m_{0}\right), \quad \varepsilon_{J}^{*}\left(l^{\prime}, m^{\prime}\right)=\varepsilon_{J}^{*}\left(\mathcal{R}^{\prime}\left(l_{0}, m_{0}\right)\right)=\varepsilon_{J}^{*}\left(l_{0}, m_{0}\right),
$$


and $\gamma_{i}\left(l^{\prime}, m^{\prime}\right)=\gamma_{i}\left(l_{0}, m_{0}\right)$. These equations can be solved uniquely. Indeed, solving the system of equations $\varepsilon_{i}\left(l^{\prime}, m^{\prime}\right)=\varepsilon_{i}\left(l_{0}, m_{0}\right), \gamma_{i}\left(l^{\prime}, m^{\prime}\right)=1\left(=\gamma_{i}\left(l_{0}, m_{0}\right)\right)(i=1, \ldots, n)$ and $\varepsilon_{i, i+1}^{*}\left(l^{\prime}, m^{\prime}\right)=$ $\varepsilon_{i, i+1}^{*}\left(l_{0}, m_{0}\right)(i=1, \ldots, n-1)$, that is, for $l^{\prime} \in \mathcal{B}_{M}$ and $m^{\prime} \in \mathcal{B}_{L}$,

$$
\begin{aligned}
& l_{i+1}^{\prime}+\frac{l_{i+1}^{\prime} m_{i+1}^{\prime}}{l_{i}^{\prime}}=\tilde{l}+\tilde{m}, \quad l_{i}^{\prime} m_{i}^{\prime}=l_{i+1}^{\prime} m_{i+1}^{\prime}, \quad i=1, \ldots, n, \\
& l_{i+2}^{\prime} m_{i+2}^{\prime}=\tilde{l} \tilde{m}, \quad i=1, \ldots, n-1,
\end{aligned}
$$

we obtain the unique solution $l_{i}^{\prime}=\tilde{m}$ and $m_{i}^{\prime}=\tilde{l}$ for any $i=1, \ldots, n+1$, which implies

$$
\left(l^{\prime}, m^{\prime}\right)=\left(m_{0}, l_{0}\right),
$$

and then $\mathcal{R}^{\prime}\left(l_{0}, m_{0}\right)=\mathcal{R}\left(l_{0}, m_{0}\right)$. According to the remark in Section 4 , we have $\mathcal{B}_{L} \cong \mathcal{V}_{L}$ and then by Corollary 3.4, $\mathcal{B}_{L} \times \mathcal{B}_{M}$ is prehomogeneous. Therefore, by Lemma 3.2 or Theorem 4.2 we have $\mathcal{R}=\mathcal{R}^{\prime}$.

Remark. We expect that this method is applicable to the tropical $\mathrm{R}$ maps of other types [3]. But we do not have explicit answers.

\section{Acknowledgments}

The author thanks the organizers of the conference "Geometric Aspects of Discrete and UltraDiscrete Integrable Systems" for their kind hospitality. He is supported in part by JSPS Grantsin-Aid for Scientific Research \#19540050.

\section{References}

[1] Berenstein A., Kazhdan D., Geometric and unipotent crystals, GAFA 2000 (Tel Aviv, 1999), Geom. Funct. Anal. (2000), Special Volume, Part I, 188-236, math.QA/9912105.

[2] Kashiwara M., Nakashima T., Okado M., Affine geometric crystals and limit of perfect crystals, Trans. Amer. Math. Soc. 360 (2008), 3645-3686, math.QA/0512657.

[3] Kashiwara M., Nakashima T., Okado M., Tropical R maps and affine geometric crystals, Represent. Theory, to appear, arXiv:0808.2411.

[4] Kuniba A., Okado M., Takagi T., Yamada Y., Geometric crystals and tropical $R$ for $D_{n}^{(1)}$, Int. Math. Res. Not. 2003 (2003), no. 48, 2565-2620, math.QA/0208239.

[5] Kac V.G., Infinite-dimensional Lie algebras, 3rd ed., Cambridge University Press, Cambridge, 1990.

[6] Kac V.G., Peterson D.H., Defining relations of certain infinite-dimensional groups, in Arithmetic and Geometry, Editors M. Artin and J. Tate, Progress in Mathematics, Vol. 36, Birkhäuser Boston, Boston, MA, 1983, 141-166.

[7] Peterson D.H., Kac V.G., Infinite flag varieties and conjugacy theorems, Proc. Nat. Acad. Sci. USA 80 (1983), 1778-1782.

[8] Kumar S., Kac-Moody groups, their flag varieties and representation theory, Progress in Mathematics, Vol. 204, Birkhäuser Boston, Boston, MA, 2002.

[9] Nakashima T., Geometric crystals on Schubert varieties, J. Geom. Phys. 53 (2005), 197-225, math.QA/0303087.

[10] Nakashima T., Geometric crystals on unipotent groups and generalized Young tableaux, J. Algebra 293 (2005), 65-88, math.QA/0403234.

[11] Nakashima T., Affine geometric crystal of type $G_{2}^{(1)}$, in Lie Algebras, Vertex Operator Algebras and Their Applications, Contemp. Math., Vol. 442, Amer. Math. Soc., Providence, RI, 2007, 179-192, math.QA/0612858.

[12] Nakashima T., Universal tropical $\mathrm{R}$ map of $\mathfrak{s l}_{2}$ and prehomogeneous geometric crystals, in New Trends in Combinatorial Representation Theory, RIMS Kôkyûroku Bessatsu, B11, Res. Inst. Math. Sci. (RIMS), Kyoto, 2009, 101-116. 